\section{CRITICAL THINKING AND CULTURE}

\section{Yoshimi Matsuda'}

The major purpose of this paper is to first argue that our thinking in general is adapted to cultural and socio-political environments. The argument will be helped by the connectionist approach that emphasizes learning and therefore nature and nurture interaction. Second, it argues that since most of our thinking or reasoning is cultural product, our evaluation of modes of thinking, too, is influenced by our familiar ways of thinking/reasoning. Third, it delineates fallibility of our cognitive processes themselves and describes some of known cognitive errors in reasoning. Finally, it discusses ways in which critical thinking may be enhanced.

\section{Culture and Thinking}

Cultural influence on thinking has been a target of inquiry for quite some time. One of the most notable attempt to explain it is the Whorfian hypothesis, although it deals specifically with linguistic determinism rather than culture in a broader sense. So far, it neither provided conclusiveevidence nor developed into a convincing theoretical framework. Recently, however, a framework that can include cultural environments as essential in our learning, and therefore thinking is getting attention in the fields of

\footnotetext{
${ }^{1}$ The School of Psychology, Australian Catholic University.
}

psychology as well as psychiatry. Although dormant for quite a while, the connectionist framework has become an increasingly popular meta-theoretical perspective on the human mind. It seems to be well poised in explaining the nature and nurture interaction and considers learning, in a broad sense, as the core of how we think, feel, and act. Learning occurs by experiencing events in one's environments, given his or her innate endowment. Thus, experience may be the engine that drives development, through channels shaped by both innate constraints and the structure of the environment. (McClelland, 1994, p.58)

This indicates plasticity of mind, nurtured in cultural and socio-political contexts. The essence of the connectionist framework of mind is to explain mental states in functionalism philosophy while closely aligning the evidence discovered in brain neurology. The connectionist framework, thus, is an attempt to connect accumulated psychological evidence with neurobiology by providing empirical support based on computer simulation. According to this framework, mind is what brain does and simultaneous activation of massively interconnected neurons in the brain perform what we normally consider thinking, while individual neurons can perform only relatively simple tasks. Information/knowledge is considered to be distributed across networked neurons rather than residing in individual neurons. Tangible knowledge is therefore materialised when a network of neurons is activated or stimulated. No executive function (acting like a homunculus) to control various subsystems of information/knowledge is proposed in the

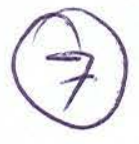


framework, and instead decision making as to which information/knowledge is used and/or combined is made by satisfying constraints inherent in individual networks representing various information/knowledge. Learning occurs in change in the ways of networks of neurons are connected, and is a continuous and slow process. We learn even when we are not intending to (i.e., the process is frequently unconscious). In this formulation, learning, thinking, and acquisition of information/knowledge are almost identical. Some involve more complicated and others simpler neural connections. Higher level thinking (such as critical thinking, reasoning, and problem solving) may be conceptualised as consisting of simultaneous activation of various networks.

Thinking, according to connectionist framework, is conceptualised in ways similar to Gestalt psychology:

thinking may involve parallel integration of knowledge based on mechanisms that are largely unconscious, sometimes producing a "restructuring" of the problem representation (Holyoak \& Spellman, 1993, p.268)

Further, similar conceptualization can be found in relationship between higherlevel thinking and sensory, perceptual, and motor processes (Holyoak et al., 1993). Connectionist as well as Gestalt frameworks contend that both higherlevel thinking and perceptual and motor processes are similar in their processing of information/knowledge. This conceptualization seems to be supported by evolutionary biology. Since the perceptual and motor processes develop- mentally precede higher-level thinking, links and therefore similarities among these processe are inevitable. This conceptualization posits that what and how we perceive strongly influences what and how we think, reason, decide, or judge. Thus, there is strong environmental influence on thinking, and the environment consists of physical as well as human made objects.

The conceptualisation of mind as a cultural and socio-political creation also comes from the discipline of cultural anthropology. For example, Fiske $(1991 ; 1992)$ used the four Social Relations Models to explain cultural construction of social behaviours and their effects on our thinking, feeling, and behaving. Four types (Communal Sharing, Authority Ranking, Equality Matching and Market Pricing) proposed by Fiske are universally available types of social interactions defining dyadic, group and institutional relations, and are used as normal standards of social interactions across cultures. Although all four forms are available in all cultures, culture also determines predominance over one form over the other. For example, currently the predominant model in the USA is said to be the Market Pricing model (Fiske et al., 1998) while that of Asia is either Communal Sharing or Authority Ranking or a combination of those (Fiske, 1991). Social relationship in the Market Pricing model is converted to monetary or otherwise tangible reward or punishment system. The Communal Sharing model is typically seen in kinship relations where members of a kin are treated equally while the Authority Ranking model places importance on hierarchy of status and power. 
Cultures which operate predominantly in Market Pricing relationships foster a strong sense of individualism.Perception of self as inter-related with others (collectivism) is fostered in the environment where Communal Sharing is predominant. Each basic social model fosters characteristic ways of thinking as well as motivational and emotional aspects of our daily lives. Each model also represents specific norms, values, and moral standards. Since these models are used not only in interpersonal (or intimate) but also group relations among individuals who may not have close contact, they encourage group as well as individually based standards of behaviour and ways of thinking. These models are therefore responsible for creating rules for resource distribution and educational and legal systems, and for ways in which these rules are enacted.

Many theorists within psychology also acknowledge the great indebtedness of our minds to social interaction. Through social interactions, we learn about "ourselves, other people, and the world" and in this sense social interaction (or communication) is the core of forming what we are, how we behave now, and how we may behave in the future (Snyder \& Stukas, 1999, p.274). Some theorists go even further contending that the tendency for thinking based on social interaction is an evolved human characteristic for survival and therefore innate. This orientation is evident in, for example, the social contract theory proposed by Cosmides (1989) and the notion of generative proclivities for social coordination by Fiske (2000). The theorization of mind as a creation of culture implies at least that cultural thinking is adaptive and needed for survival within a particular community.

The relevant literature generally indicates it is widely accepted that cultural and the social system influences thinking and that a particular cultural mode of thinking represents the well-adopted model to the context. These arguments often lead to grudging acceptance that evaluation of a cultural model is not legitimate without taking the context into consideration. A question of superiority, nevertheless, may arise, for example, in terms of creative thinking and reasoning suitable for technological and scientific advancement and therefore economic benefits. This may be particularly so in postindustrial society where "Scientific-theoretical knowledge, accordingly, becomes a major force of economic growth and social progress" (Szelenyi \& Martin, 1991, p.24)

A conventional argument is that the analytic mode of thinking prevalent in the West brought great advantages in scientific advancement and the current economic prosperity of the western nations. This, however, ignores their long-standing colonization history of Asia and Africa and residual benefits since then. In educational literature, too, the East has been frequently compared with the West: rote learning method of the East has often been pitted against analytical thinking of the West, and, in spite of some contradictory evidence (Matheis, Spooner, Coble, Takemura, Matsumoto, Matsumoto, \& Yoshida, 1992) and baffling superior achievement of supposedly rote learners (Suter, 1988), the myth still continues. In the English language research literature, evaluation of supe- 
riority or inferiority of one thinking mode over others, whether it is about school achievement, economic gain, morality, values or scientific discovery, is often tainted by ethnocentrism of English-speaking culture. Since the emergence and subsequent rapid development of electronic communication systems such as internet, English language dominance over world communication has increased and thus also the influence of Anglo-American perceptions. Asians must be vigilant not to be swept away by such influence, as in the analysis of the Asian financial crisis when cultural factors such as corrupt business practices were blamed rather than the possible consequences of emerging new global financial systems to which the West were more adept since they are modeled after western tradition (Johnson, 2000). Arguments of superiority are frequently thinly disguised as arguments of power: whoever holds power can dictate what is superior.

Research on analytic versus holistic ways of thinking have been well documented comparing the so-called Eastern (e.g., China and Japan) and Western (e.g., the USA and the Western European countries) cultural groups. Resurgence of inquiry into cultural difference in thinking is evident in recent psychological literature. Some conducted jointly by researchers from both areas. Review of some cross-cultural research (Fiske, Kitayama, Markus, \& Nisbett, 1998) indicates that Westerners tend (1) to study objects/entities by breaking them into component elements, (2) to reason about their characteristics "in linear, deterministic fashion (p.934), and therefore (3) to place objects/entities in categories. These characteristics are said to be generally absent in Eastern thinking. People in the East tend to study objects/entities as a whole, to understand their characteristics in non-linear and non-deterministic fashion, and to see no particular worth in categorization. Western style deductive reasoning (e.g., A and not-A cannot both be true, (p.934)) is also said to be missing in Eastern thinking. Instead, Eastern thinking is dialectical and assumes precisely that both A and not-A can be true, indeed that $\mathrm{A}$ implies the existence, or the immanent existence, of not-A (p.934)

Some theorists consider dialectical thinking to be more mature in comparison with other modes of thinking (Kramer \& Melchior, 1990).

In Western theories of psychology, the characteristics of thinking delineated above are clearly evident. Recently, however, a shift toward more non-linear, non-deterministic models of human behavior is emerging in the field reflecting the sterile effects brought by linear and deterministic models of human behavior. One such example is the connectionist orientation mentioned earlier. While the East has been beguiled by affluence of the West and eager to learn Western styles, some Western thinkers gained insights into Eastern ways of thinking particularly in business and technology areas during the ascent of Asian "tiger economies" in the 1980s. The progress toward more equitable evaluation of cultural ways, either East, West or other, seemed to be set aback in the aftermath of the Asian financial crisis, suggesting to some that the West is 
better after all. The emotional nature of cultural evaluation may be inevitable in international competition (this coincidently points to the interaction between emotion and cognition, and to contextual influence on thinking), but learning the advantages and disadvantages of world cultures seems to be one of the important components for enhancing critical thinking. Sometimes learning fostered in culture hinders and sometimes enhances flexibility of thinking. Cultures are known to be different not only in the above mentioned dimensions of thinking but also other aspects such as moral reasoning and causal attribution.Some studies of moral reasoning report the tendency of some Asian cultures to reason based on obligation based relationships in comparison with the Westerners (Miller, 1994). Studies comparing the attribution style between the East and West show that the people in the East consider situational factors as important in making causal attribution in general (Choi, Nisbett, \& Norenzayan, 1997). On the other hand, the Westerners tend to infer personality factors in the situation not involving oneself. Moral reasoning and causal attribution play important roles in business transactions, governmental policies, international relations, pedagogies, and priorities placed on resource allocation to name a few.

\section{Critical Thinking}

What the term, critical thinking, actually means is controversial other than that it belongs to the category of higherlevel thinking. Some differentiate criti$\mathrm{cal}$ thinking from creative thinking. To those who differentiate, critical think- ing consists of ways to detect flaws and mistakes in existing ideas or solutions while creative thinking leads to new solutions and ideas. However, that critical thinking can also bring about creative thinking is evident.

Further conceptual complication may emerge in terms of the relation between thinking and feeling, and various other categorizations of mental activities. Contemporary literature on cognition claims a bi-directional influence between feeling and thinking. The usual distinction between hot and cold cognition is diffused. A distinction such as social cognition versus pure cognition is not very meaningful, either. Although the distinction between deductive (topdown processing) versus inductive (bottom-up processing) still exists and is widely used, close scrutiny reveals ambiguity in directionality imposed in the differentiation: both inferential processes seem to use top-down and bottom-up reasoning in the process of obtaining inferences some times (Kurtz, Gentner, \& Gunn, 1999). Some propose, therefore, that differentiation may be more appropriate in terms of weak versus strong reasoning methods. Weak reasoning method relies on content neutral syntactic rules while strong reasoning heavily utilizes experiences and available knowledge representation (Kurtz et al., 1999). The inferential or reasoning process is better understood in a continuum from weak to strong methods. Both methods are important in successful thinking.

Critical thinking, if understood broadly, requires both generation and evaluation of beliefs/ideas. Generation and evalua- 
tion, in turn, require holistic thinking (Holyoak et al., 1993) and they are essentially products of reasoning. Some contend that success in reasoning resides more in "the relationship between the content of the premises and prior knowledge than ... the logical form of the reasoning involved" (Holyoak et al., 1993, p.292)

In the process of reasoning, we go beyond initially available (or presented) information and, as an end result, produce inferences. Thus, reasoning underlies most of our mental processes such as "the expectations, generalizations, and assertions people reach in interpreting events and situations" (Kurtz, et al., 1999, p. 145). Reasoning itself may be considered the combination of basic components: initial information, stored relevant knowledge, the cognitive process used (i.e., inductive, deductive, analytic, holistic, flexible, stereotypic thinking, etc.), and generated inferences themselves. This formulation of reasoning indicates that how original information is represented is important, and how it is represented may depend on the depth and variety of the domain knowledge. Which cognitive process is predominantly used may also depend on the representation of the original information. This view of reasoning linking stored knowledge (and therefore memory traces) leads further to the link with learning and acquisition of knowledge. Thus, Differences in reasoning performance with learning and development or across domains can be linked to conceptual change and variation in the amount, nature, and structure of the available knowledge (Kurtz, et al., 1999, p. 146).
Other research also indicates the importance of how reasoning content is represented in the success of reasoning. For an inferential process to be successful, the information should be represented in a definite, therefore vivid rather than vague and pallid, manner. Holyoak and Spellman (1993) summarise the research.

In a vivid representation, a finite number of objects are represented and each is associated with definite values for all relevant properties and relation. (p.301).

And in general, a vivid representational system is one that compels specification of certain information and that specifies interdependencies between properties and relations so that a partially specified input can yield a definite model. In such a system, to think a certain thought may not only entail that you can think some other related thought .... but that you are thinking it. (p.302).

The concept of vivid representation is feasible not only in terms of spatial imagery but also in terms of other cognitive representation within the connectionist model as well as by the schema model of information processing. All these are considered to be able to possess characteristics of vivid representation. Vivid representation of information does not mean concreteness only. It also facilitates abstraction. For example, schemas guide us into creating possible models of solutions and decision-making. Once feasible models are constructed, each piece of information can be evaluated to be either relevant or irrelevant to the task at hand. The importance of vivid representation 
is known not only in thinking but also in memory itself. Since our working memory is limited in capacity, only a small amount of information can be dealt with at one time. Thus, the small amount of vividly represented information enhances our reasoning process.

Research on experts also indicates the importance of stored knowledge and knowledge representation. Experts are different from novices generally in the following aspects: (1) memory for relevant information presented, (2) important and relevant knowledge of the target domain(s), (3) problem representations in terms of appropriate mental images, diagrams, etc., (4) finding structural similarities between problems, (5) finding constraints of problem, (6) speed and efficiency, and (7) monitoring problem solving - judging difficulty of problem, being aware of making errors, more appropriately allocating time for solving problems (Matlin, 1994).

Experts or expertise, however, come in two types: routine versus adaptive. Routine expertise is characterized by quick and accurate solution produced for well-practiced problems, while adaptive expertise is able to utilize knowledge in novel situations. The key to adaptive expertise is considered to be deep and explicit understanding of the target domain (Hatano \& Inagaki, 1986). In other words, deep and explicit understanding leads to vivid representation.

At the very foundation, however, critical thinking requires guarding against habitual ways of thinking such as mental sets (e.g., Luchin's (1942) Water-Jar problems indicating rigid use of problem solving techniques) and functional fixedness (rigid thinking in assigning objects' functions). In addition to these inherent pitfalls to our reasoning, other elements are known to lead us astray in our judgment and decision making processes. Our reasoning/thinking is heavily influenced by socio-cultural contexts. Some of these elements are briefly discussed below, based on Plous (1993).

Selective perception - we tend to see what we expect to see. Our sensory perception is also our reconstruction of reality and is dependent upon our cognitive and motivational orientation. Topdown methods of reasoning guided by expectation tends to leads us to erroneous conclusions.

Cognitive dissonance - we are motivated to reduce psychological inconsistencies. We feel uncomfortable holding two contradictory or inconsistent thoughts and in the process of reducing the inconsistencies, we come to illogical conclusions.

Biases in memory - contents of memory depends on the particular situation when that memory is recalled. The contents of memory do not stay the same and they are reconstructed each time we remember. Thus, memory based judgment should be treated with caution.

Context dependent nature of perception - our perception is easily influenced by the context in which objects or events are placed. Some specific context effects that are considered particularly important in decision making and judgment are the contrast effect (e.g., after lifting a heavy weight, a relatively heavy 
weight lifted next tends to be perceived as lighter than it actually is), the primacy effect (e.g., the first impression influences the second and third impressions of objects and events), the recency effect (e.g., the final evaluation of objects or events has strong and undue influence on judgment), and halo effects (e.g., evaluation of one aspect influences evaluation of another that should logically not be related).

The representativeness heuristic (general rule of thumb) - our evaluation and judgment heavily rely on characteristic similarities among events. This tendency further brings about preference for detailed causal reasoning that does not reflect reality or judgment that the probability of two events occurring simultaneously is greater than the probability of a single event. For example, "the defendant left the scene of the crime may appear less plausible than ... the defendant left the scene of the crime for fear of being accused of murder." (p.111)

The availability heuristic - we tend to make decisions by assessing "the frequency of a class or the probability of an event by the ease with which instances or occurrences can be brought to mind" (p.121).This type of mental shortcut also utilizes the use of imagination in causal inferences. For example, we tend to get more upset knowing a bus was delayed and that it just departed than the case where the bus left as scheduled. This is presumably because we can think of lots reasons why we might have been able to catch the bus in the first case.
There are more and they can be easily identified in the literature. They are considered to be operative in any culture, although the precise situation in which each of the above takes place is culture specific. For example, what two events are considered similar or which instances or occurrences can be brought to mind with ease depends on the cultural context. Heuristics are useful for simplifying an otherwise complicated reasoning process, but heavy reliance on them usually results in unsuccessful reasoning.

We should be aware of these possible pitfalls and weaknesses in our reasoning process. These can be easily taught to students along with cross-cultural aspects in a formal educational system. At the same time, teacher education should also clearly emphasize basic and universal components as well as crosscultural aspects of reasoning.

The importance of educating people in reasoning is also indicated by the study of expertise. Deep and explicit understanding is more likely to occur in the learning environment where learning task is "variable and in some degree unpredictable rather than stereotyped, and when the task is explored freely without heavy pressure to achieve an immediate goal" (Sweller, 1988 cited in Holyoak et al., 1993, p.298). These research results seem to point to the importance of the educational environment that encourages exploration in depth and variety without undue pressures, instills joy of learning and is democratic. In this educational environment, learning various cultural modes of thinking should also be encouraged as one of the 
ways to advance adaptive expertise, and therefore critical thinking. Understanding cross-cultural differences and similarities is important for the reduction of ethnocentric and stereotypical thinking that hampers any advancement.

\section{Critical Thinking in Contexts}

Scientific and technological knowledge and development may flourish under a non-democratic regime with strong pressure for such development as we have seen in the Nazi and Stalinist regimes. Indoctrinating ideologies of the regime, however, do not encourage innovative thoughts that deviate from doctrine, thus eventually drying up creativity. On the other hand, there are ethical and moral obligations on the part of those who are involved in the pursuit of knowledge and development of science and technology. Their goals should be for the benefit of humanity and not only for the pursuit of profits for an elite or development for the sake of development. These concerns are in the realm of civic culture and are also educational concerns; not only formal education but also continuing education throughout one's life. According to Dewey (1916), "education means the enterprise of supplying the conditions which insure growth, or adequacy of life, irrespective of age" (p.61) and "The inclination to learn from life itself and to make the conditions of life such that all will learn in the process of living is the finest product of schooling." (p.60).

Thus, the importance of education is not only in teaching particular knowledge but also in instilling the enthusiasm to learn. The latter cannot be realised in a community without freedom from coercion and economic security. Given a community with such basic conditions, the first step toward this end seems to start with quality of teacher education and quality of applicants who aspire to be teachers. The community should be able to attract the talented to the field of education, and at the same time the institution for training teachers should include the community's best scholars in each substantive field. But these are not sufficient conditions. Since education itself requires sound knowledge of behavioral sciences such as cultural anthropology, psychology, and sociology, resource distribution of the community cannot favor only the so-called hard science and technology. Given also that critical evaluation and generation of new ideas rest on variety as well as depth of knowledge, recent policies regarding governmental funding biased toward business, science and technology to the exclusion of philosophy, language, and arts are misconstrued. This exclusion model of resource distribution tacitly acknowledges hierarchies of academic disciplines and in turn may determine attraction only to these fields. This is a tragedy not only in terms of science and technology development per se but also their development toward a humane direction.

The above arguments advocate a civic culture where diversity of opinion/thinking is the norm and encouraged: where different opinions are freely exchanged without fear of censorship provided such opinions do not preach the destruction of civic society. Such civic culture should also encourage diversity of mass media outlets and take responsibility for 
providing access to different ways of reasoning and thinking for its constituents. Families and communities that participate in the rich intellectual communication resources provided in such culture encourage their children and adults in the life-long pursuit of knowledge without uncritical acceptance of the status quo.

\section{References}

Choi, I., Nisbett, R. E., \& Norenzayan, A. (1997). Culture, category salience, and inductive reasoning. Cogni tion, 65, 15-32.

Cosmides, L. (1989). The logic of social exchange: Has natural selection shaped how humans reason? Stud ies with the Wason selection task. Cognition, 31, 187-276.

Dewey, J. (1916). Democracy and educa tion. New York: Mcmillan.

Fiske, A. P. (2000). Complementarity theory: Why human social capacities evolved to require cultural comple ments. Personality and Social Psy chology Review, 4, 76-94.

Fiske, A. P., Kitayama, S., Markus, H. R., \& Nisbett, R. E. (1998). The cultural matrix of social psychology. In D. T. Gilbert, S. T. Fiske, \& G. Lindzey (Eds.), Handbook of so cial psychology (4 ${ }^{\text {th }}$ ed., Vol. 2, pp.915-981). New York: Oxford University Press.

Hatano, G. \& Inagaki, K. (1986). Two courses of expertise. In $\mathrm{H}$. Stevenson, H. Azuma, \& K. Hakuta (Eds.), Child development and edu cation in Japan (pp. 262-272). San Francisco: Freeman.
Holyoak, K. J. \& Spellman, B. A. (1993). Thinking. Annual Review of Psychology, 44, 265-315.

Johnson, C. (2000). Blowback: The cost and consequences of American empire. New York: Metropolitan Books.

Kramer, D. A., \& Melchior, J. (1990). Gen der, role conflict, and the develop ment of relativistic and dialectical thinking. Sex Roles, 23, 553-575.

Kurtz, K. J., Gentner, D., \& Gunn, V. (1999). Reasoning. In B. M. Bly \& D. E. Rumelhart (Eds.), Cognitive science (pp. 145-200). San Diego: Academic Press.

Luchins, A. S. (1942). Mechanization of problem solving. Psychological Monographs, 54 (whole No. 248).

Matheis, F. E. \& Spooner, W. E., Coble, C. R., Tekemura, S., Matsumoto, S., Matsumoto, K., \& Yoshida, A. (1992). A study of logical think ing skills and integrated process skills of junior high school students in North Carolina and Japan. Science Education, 76, 210-222.

Matlin, M. W. (1994). Cognition ( $3^{\text {rd }}$ ed.) Fort Worth, TX: Harcourt Brace Publisher.

Miller, J. G. (1994). Cultural diversity in the morality of caring: Individually oriented versus duty-based inter personal moral codes. Cross-Cul tural Research, 28, 3-39.

Plous, S. (1993). The psychology of judg ment and decision making. New York: McGraw-Hill.

Plous, S. (1993). The psychology of judg ment and decision making. New York: McGrow-Hill. 
Suter, L. E. (1988). Selected indicators of education of the United States and Japan: An application of the Cen ter for Education Statistics outline of indicators to cross national com parison. Annual Meetings of the American Educational Research Association, New Orleans, LA, USA.

Szelenyi, I. \& Martin, B. (1991). The three waves of new class theories and a postscript. In C. C. Lemert (Ed.), Intellectuals and politics: Social theory in a changing world. Newbury Park, CA: Sage.

Wallace-Bruce, N. L. (2000). Corruption and competitiveness in global business - the dawn of a new year.

Melbourne University Law Review, 24, 349-378. 